\title{
Global exponential stabilization of delayed BAM neural networks: a matrix measure approach
}

\author{
Yong Li, Guodong Wu, Liu Peng, Guangming Cao \\ Wuhan Second Ship Design and Research Institute, Wuhan, China \\ e-mail: liyonglynn@163.com
}

Keywords: global exponential stabilization; BAM neural networks; time delays; matrix measure; Lyapunov stability theory

\begin{abstract}
In this paper, global exponential stabilization of bidirectional associative memory (BAM) neural networks with time delays is investigated. Based on the Lyapunov stability theory, we present several sufficient conditions for the global exponential stability of the equilibrium point of the BAM neural networks with delays via matrix measure approach. The presented results are easy to verify and simple to implement in practice. Finally, one numerical example is given to illustrate the feasibility and effectiveness of our theoretical results.
\end{abstract}

\section{Introduction}

Bidirectional associative memory (BAM) neural networks are the minimal two-layer nonlinear feedback network and behave as a two-layer hierarchy of symmetrically connected neurons, which were first proposed by Kosko [1,2].This model is an important network because of its ability of information memory and information association. Therefore, BAM neural networks have crucial application prospect in pattern recognition and automatic control engineering. Owing to the unavoidable finite signal transmission time and switching speed, the occurrence of time delay is ubiquitous and inevitable in nature. Then, the analysis of the delayed BAM neural networks has received considerable attention; especially, dynamical behaviors of delayed BAM neural networks have been extensively investigated. It can be noticed that most of the existing works focus on the stability analysis [3, 4], synchronization problem [5], periodic oscillation [6, 7], optional problem [8, $9]$, or bifurcation [10,11]. In [3], a new robust stability was studied for a class of uncertain neutraltype neural networks with discrete and distributed delays. In [5], global exponential synchronization of delayed BAM neural networks was investigated by using Lyapunov stability theory and Halanay inequality. In [7], the existence of periodic solutions in a six-neuron BAM network model by means of Chafee's criterion of limit cycle was discussed. In [11], bifurcation and control in a neural network with small and large delays was investigated.

In this paper, a novel approach based on matrix measure is introduced to investigate the stabilization of the delayed BAM neural networks. The matrix measure approach has several advantages as follows: firstly, different from the previous methods, we can directly use the matrix measure representation of state or error vector as the Lyapunov function or functional without elaborate structure. Besides, the matrix measure can trade off the effects of the negative values as well as the positive values of the matrix, while noting that most of the previous results on stability or synchronization are in form of norm, which can only be nonnegative values and limit the scope of nonnegative values. Thus, this method is more powerful and precise when dealing with the case when the connection weights contain both positive and negative values, which can correspond to the excitation and inhibition in neurons, respectively. In general, the matrix measure method utilizes the information of matrix elements, especially the diagonal elements of a matrix more sufficiently, so in some cases it has broader applications. It can be found that more and more papers have applied the matrix measure approach [12-15]. In [13], a single inertial BAM neural network with time-varying delays and external inputs is concerned with matrix measure strategies. In [14], synchronization 
problem of switched interval delayed networks under the arbitrary switching rule by intermittent control was studied based on matrix measure theory.

Inspired by the above analysis, this paper mainly focuses on the exponential stabilization of a class of BAM neural networks with time delays. Different from the past studies, a novel approach based on matrix measure and Halanay inequality is introduced and we obtain several simple but generic conditions for the global exponential stability of the equilibrium point under feedback control. Compared with the previous results, in our theoretical results requirement of the controller gain matrices is less restrictive and neither symmetry nor positive (negative) definiteness of the coupling matrix is required. It has shown that the sufficient conditions for the exponential stabilization are easy to verify. And it only needs to adjust the controller gain matrices to satisfy the sufficient conditions obtained; then, stabilization of BAM neural networks will be achieved.

The rest of this paper is organized as follows. In Section 2, we describe the delayed BAM neural networks considered in this paper, define matrix measure, and present some necessary preliminaries. In Section 3, by applying the matrix measure approach and Lyapunov stability theory, global exponential stability of the equilibrium point is investigated. In Section 4, an illustrative example is given to validate the effectiveness and feasibility of the obtained results and the developed method. Finally, in Section 5, the conclusions are drawn.

\section{Systems description and preliminaries}

A class of BAM neural networks with time delays considered in this paper is described by the following delayed differential equations:

$$
\left\{\begin{array}{l}
\dot{x}_{i}(t)=-c_{i} x_{i}(t)+\sum_{j=1}^{n} w_{j i} f_{j}\left(y_{j}(t-\tau)\right)+I_{i}, i=1,2, \ldots, m, \\
\dot{y}_{i}(t)=-d_{j} y_{j}(t)+\sum_{i=1}^{m} h_{i j} g_{i}\left(x_{i}(t-\sigma)\right)+J_{j}, j=1,2, \ldots, n,
\end{array}\right.
$$

where $x_{i}(t)$ and $y_{j}(t)$ are the state variables of the ith neuron and the jth neuron, respectively; $c_{i}$ and $d_{j}$ are appropriately selected constants such that the solution of the model given in (1) remains bounded; time delays $\tau$ and $\sigma$ are both nonnegative constants, which correspond to the transmission delays of the neurons. $w_{j i}$ and $h_{i j}$ are the connection weights, which denote the strengths of connectivity between the neuron $i$ and neuron $j$ at time $t-\tau$ and $t-\sigma$, respectively; $f_{j}, g_{i}$ are inspirit functions, which describe the manner in which the neurons respond to each other with $f(0)=0, g(0)=0 . I_{i}, J_{j}$ denote constant external input vectors of the ith neuron and the jth neuron introduced from outside the network, respectively.

If we denote

$$
\begin{aligned}
& x(t)=\left[x_{1}(t), x_{2}(t), \ldots, x_{m}(t)\right]^{T} ; \\
& y(t)=\left[x_{1}(t), y_{2}(t), \ldots, y_{n}(t)\right]^{T} ; \\
& C=\operatorname{diag}\left(c_{1}, c_{2}, \ldots, c_{m}\right), D=\operatorname{diag}\left(d_{1}, d_{2}, \ldots, d_{n}\right) ; \\
& W=\left(w_{j i}\right)_{m \times n}, H=\left(h_{i j}\right)_{n \times m} ; \\
& f(y(t-\tau))=\left[f_{1}\left(y_{1}(t-\tau)\right), f_{2}\left(y_{2}(t-\tau)\right), \ldots, f_{n}\left(y_{n}(t-\tau)\right)\right]^{T} ; \\
& \quad g(x(t-\sigma))=\left[g_{1}\left(x_{1}(t-\sigma)\right), g_{2}\left(x_{2}(t-\sigma)\right), \ldots, g_{m}\left(x_{m}(t-\sigma)\right)\right]^{T} ; I_{i}=\left[I_{1}, I_{2}, \ldots, I_{m}\right]^{T}, J_{j}=\left[J_{1}, J_{2}, \ldots, J_{n}\right]^{T} ;
\end{aligned}
$$

Then, system (1) can be rewritten in the following vector-matrix form:

$$
\left\{\begin{array}{l}
\dot{x}(t)=-C x(t)+W f(y(t-\tau))+I \\
\dot{y}(t)=-D y(t)+H g(x(t-\sigma))+J .
\end{array}\right.
$$

The initial conditions of system (2) are given by $x(s)=\varphi_{x}(s), s \in[-\sigma, 0], y(s)=\varphi_{y}(s), s \in[-\tau, 0]$, in which $\varphi_{x}(s)$ and $\varphi_{y}(s)$ are continuous functions. 
Definition 1 The point $\left[x^{*}, y^{*}\right]^{T}$ with $x^{*}=\left[x_{1}^{*}, x_{2}^{*}, \ldots, x_{m}^{*}\right]^{T} y^{*}=\left[y_{1}^{*}, y_{2}^{*}, \ldots, y_{n}^{*}\right]^{T}$ is called an equilibrium of system (2) if

$$
\left\{\begin{array}{l}
-C x^{*}+W f\left(y^{*}\right)+I=0 \\
-D y^{*}+H g\left(x^{*}\right)+J=0
\end{array}\right.
$$

$\|\varphi\|_{p}=\sup _{t_{0}-\tau \leq s \leq t_{0}}\|\varphi\|_{p}(s)$ is used to denote the norm of a function $\varphi \in C\left(\left[t_{0}-\tau, t_{0}\right], R^{n}\right) .\|\cdot\|_{p}$ is a vector norm and $p=1,2, \infty$. For $x \in R^{n}$, the vector norm $\|\cdot\|_{p}$ is defined as

$$
\|x\|_{1}=\sum_{i=1}^{n}\left|x_{i}\right|, \quad\|x\|_{2}=\sqrt{\sum_{i=1}^{n} x_{i}^{2}},\|x\|_{\infty}=\max _{1 \leq i \leq n}\left|x_{i}\right| .
$$

With regard to functions $f(\cdot)$ and $g(\cdot)$, we have the following assumption:

Assumption 1 Each function $f_{j}: R \rightarrow R, j=1,2, \ldots, n$ and $g_{i}: R \rightarrow R, i=1,2, \ldots, m$ are bounded and satisfy the Lipschitz conditions with Lipschitz constants $K>0, L>0$, i.e.,

$$
\begin{gathered}
|f(u)-f(v)| \leq K|u-v|,|g(u)-g(v)| \leq L|u-v| \\
\text { for all } u, v \in R .
\end{gathered}
$$

Now, we introduce the concept of matrix measure.

Definition 2[16] The matrix measure of a real square matrix $A=\left(a_{i j}\right)_{n \times n}$ is as follows:

$$
\mu_{p}(A)=\lim _{\varepsilon \rightarrow 0^{+}} \frac{\|I+\varepsilon A\|_{p}-1}{\varepsilon},
$$

where $\|\cdot\|_{p}$ is an induced matrix norm on $R^{n \times n}, I$ is the identity matrix, and $p=1,2, \infty$.

When the matrix norm

$$
\|A\|_{1}=\max _{j} \sum_{i=1}^{n}\left|a_{i j}\right|, \quad\|A\|_{2}=\sqrt{\lambda_{\max }\left(A^{T} A\right)}, \quad\|A\|_{\infty}=\max _{i} \sum_{j=1}^{n}\left|a_{i j}\right|,
$$

we can obtain the matrix measure

$$
\mu_{1}(A)=\max _{j} a_{j j}+\sum_{i=1, i \neq j}^{n}\left|a_{i j}\right|, \quad \mu_{2}(A)=\frac{1}{2} \lambda_{\max }\left(A^{T}+A\right), \quad \mu_{\infty}(A)=\max _{i}\left\{a_{i i}+\sum_{j=1, j \neq i}^{n}\left|a_{i j}\right|\right\}
$$

Lemma 1[17] Let $k_{1}$ and $k_{2}$ be constants with $k_{1}>k_{2}>0$, and $y(t)$ is a nonnegative continuous function defined on $\left[t_{0}-\tau,+\infty\right]$, which satisfies the following inequality for $t \geq t_{0}$ :

$$
D^{+} y(t) \leq-k_{1} y(t)+k_{2} \bar{y}(t)
$$

where $\bar{y}(t)=\sup _{t-\tau \leq s \leq t} y(s)$. Then

$$
y(t) \leq \bar{y}\left(t_{0}\right) e^{-\lambda\left(t-t_{0}\right)},
$$

where $\lambda>0$ is the unique positive solution of $\lambda=k_{1}-k_{2} e^{\lambda \tau}$, here the upper right Dini derivative $D^{+} y(t)$ is defined as

$$
D^{+} y(t)=\varlimsup_{h \rightarrow 0^{+}} \frac{y(t+h)-y(t)}{h},
$$

where $h \rightarrow 0^{+}$means that $h$ approaches 0 from the right hand side.

Throughout the proofs of the Theorems in this paper, Taylor's formula with remainder of Peano is applied; that is, $x(t+h)=x(t)+h \dot{x}(t)+o(h)$, where $o(h)$ denotes high-order infinitesimal of $h$.

\section{GLOBAL EXPONENTIAL STABILITY OF THE EQUILIBRIUM POINT}

In this section, we shall establish several sufficient conditions in the form of matrix measure to ensure the global exponential stability of the equilibrium point of system (2) based on the Lyapunov stability theory.

Here, suppose that $\left[x^{*}, y^{*}\right]^{T}$ is the equilibrium point of system (2) and will obtain our conditions when the equilibrium point of system (2) is at the origin, that is $\left[x^{*}, y^{*}\right]^{T}=[0,0]^{T}$. There is no loss 
of generality in doing this because any equilibrium point can be shifted to the origin. The translations $u(t)=x(t)-x^{*}, v(t)=y(t)-y^{*}$ transform system (2) into the following system:

$\left\{\begin{array}{l}\dot{u}(t)=-C u(t)+W \bar{f}(v(t-\tau)), \\ \dot{v}(t)=-D v(t)+H \bar{g}(u(t-\sigma)),\end{array}\right.$

where $u(t)$ and $v(t)$ are the state vectors of the transformed system and

$$
\bar{f}(v(t-\tau))=f(y(t-\tau))-f\left(y^{*}\right), \bar{g}(u(t-\sigma))=g(x(t-\sigma))-g\left(x^{*}\right) .
$$

Note that the functions $\bar{f}(\cdot)$ and $\bar{g}(\cdot)$ satisfy the assumptions (i.e., Assumption 1) on $f(\cdot)$ and $g(\cdot)$. Therefore, we assume that the vector $[I, J]^{T}=[0,0]^{T}$ in (2) and study the stability properties of the origin of the following system:

$$
\left\{\begin{array}{l}
\dot{x}(t)=-C x(t)+W f(y(t-\tau)), \\
\dot{y}(t)=-D y(t)+H g(x(t-\sigma)) .
\end{array}\right.
$$

In the following, sufficient condition is presented for the exponential stability of the equilibrium point of the system defined by (3).

In order to stabilize the origin of the system (3), a control law will be designed. The controlled system can be described by the following differential equation:

$$
\left\{\begin{array}{l}
\dot{x}(t)=-C x(t)+W f(y(t-\tau))-r(t) \\
\dot{y}(t)=-D y(t)+H g(x(t-\sigma))-s(t)
\end{array}\right.
$$

where $r(t)$ and $s(t)$ denote the control input vectors and

$$
r(t)=\left[r_{1}(t), r_{2}(t), \ldots, r_{m}(t)\right]^{T}, s(t)=\left[s_{1}(t), s_{2}(t), \ldots, s_{n}(t)\right]^{T} .
$$

And, we assume that the control imposed on system (4) is of the following form:

$$
\left[\begin{array}{c}
r_{1}(t) \\
\vdots \\
r_{m}(t)
\end{array}\right]=R\left[\begin{array}{c}
x_{1}(t) \\
\vdots \\
x_{m}(t)
\end{array}\right]=R x(t),\left[\begin{array}{c}
s_{1}(t) \\
\vdots \\
s_{n}(t)
\end{array}\right]=S\left[\begin{array}{c}
y_{1}(t) \\
\vdots \\
y_{n}(t)
\end{array}\right]=S y(t),
$$

in which $R=\left(r_{j i}\right)_{n \times m}$ and $S=\left(s_{i j}\right)_{m \times n}$ are the controller gain matrices.

Theorem 1 Under Assumption 1, if controller gain matrices $R, S$ satisfy

$$
\left.-\mu_{p}\left(\begin{array}{cc}
(-C-R) & 0 \\
0 & (-D-S)
\end{array}\right]\right)>\max \left(K\|W\|_{p}, L\|H\|_{p}\right)>0
$$

Then, the origin of system (3) is globally exponentially stable, $p=1,2, \infty$.

Proof. Definite $\Lambda(t)=\left[\begin{array}{ll}x(t) & y(t)\end{array}\right]^{T}$, then, let us consider the following Lyapunov function:

$$
V(t)=\|\Lambda(t)\|_{p}=\left\|\left[\begin{array}{l}
x(t) \\
y(t)
\end{array}\right]\right\|_{p} .
$$

First, the upper right derivative of $V(t)$ with respect to time $t$ along the system (4) is as follows:

$$
\begin{aligned}
& D^{+} V(t)=\varlimsup_{h \rightarrow 0^{+}} \frac{\|\Lambda(t+h)\|_{p}-\|\Lambda(t)\|_{p}}{h} \\
& =\varlimsup_{h \rightarrow 0^{+}} \frac{\|\Lambda(t)+h \dot{\Lambda}(t)+o(h)\|_{p}-\|\Lambda(t)\|_{p}}{h} \\
& \leq \varlimsup_{h \rightarrow 0^{+}} \frac{\left\|\Lambda(t)+h\left[\begin{array}{c}
\dot{x}(t) \\
\dot{y}(t)
\end{array}\right]\right\|_{p}-\|\Lambda(t)\|_{p}}{h}
\end{aligned}
$$

Substituting equality (4) into the right side of equality(6) yields

$$
D^{+} V(t) \leq \varlimsup_{h \rightarrow 0^{+}} \frac{\left\|\left[\begin{array}{l}
x(t) \\
y(t)
\end{array}\right]+h\left[\begin{array}{c}
-C x(t)+W f(y(t-\tau))-r(t) \\
-D y(t)+H g(x(t-\sigma))-s(t)
\end{array}\right]\right\|_{p}-\|\Lambda(t)\|_{p}}{h}
$$




$$
\begin{aligned}
& \leq \varlimsup_{h \rightarrow 0^{+}} \frac{\left\|\left[\begin{array}{l}
x(t) \\
y(t)
\end{array}\right]+h\left[\begin{array}{l}
-C x(t)+W f(y(t-\tau))-R x(t) \\
-D y(t)+H g(x(t-\sigma))-S y(t)
\end{array}\right]\right\|_{p}-\|\Lambda(t)\|_{p}}{h} \\
& =\varlimsup_{h \rightarrow 0^{+}} \frac{\left\|\left[\begin{array}{l}
x(t) \\
y(t)
\end{array}\right]+h\left[\begin{array}{l}
(-C-R) x(t)+W f(y(t-\tau)) \\
(-D-S) y(t)+H g(x(t-\sigma))
\end{array}\right]\right\|_{p}-\|\Lambda(t)\|_{p}}{h} \\
& \leq \varlimsup_{h \rightarrow 0^{+}} \frac{\left\|\left[\begin{array}{l}
x(t) \\
y(t)
\end{array}\right]+h\left[\begin{array}{l}
(-C-R) x(t) \\
(-D-S) y(t)
\end{array}\right]\right\|_{p}-\|\Lambda(t)\|_{p}}{h}+\left\|\left[\begin{array}{l}
W f(y(t-\tau)) \\
H g(x(t-\sigma))
\end{array}\right]\right\|_{p}
\end{aligned}
$$

Based on Assumption 1 and $f(0)=0, g(0)=0$, we have

$$
\|f(y(t-\tau))\|_{p} \leq K\|y(t-\tau)\|_{p},\|g(x(t-\sigma))\|_{p} \leq L\|x(t-\sigma)\|_{p} .
$$

Then, we can obtain

$$
\begin{aligned}
& D^{+} V(t) \leq \varlimsup_{h \rightarrow 0^{+}} \frac{\left\|\left[\begin{array}{l}
x(t) \\
y(t)
\end{array}\right]+h\left[\begin{array}{l}
(-C-R) x(t) \\
(-D-S) y(t)
\end{array}\right]\right\|_{p}-\|\Lambda(t)\|_{p}}{h}+\left\|\left[\begin{array}{l}
K W y(t-\tau) \\
L H x(t-\sigma)
\end{array}\right]\right\|_{p}
\end{aligned}
$$

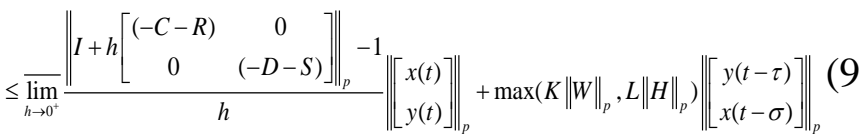

Based on Definition 1, we have

$$
\begin{aligned}
& \left.D^{+} V(t) \leq \mu_{p}\left(\begin{array}{cc}
(-C-R) & 0 \\
0 & (-D-S)
\end{array}\right]\right)\left\|\left[\begin{array}{l}
x(t) \\
y(t)
\end{array}\right]\right\|_{p}+\max \left(K\|W\|_{p}, L\|H\|_{p}\right)\left\|\left[\begin{array}{c}
y(t-\tau) \\
x(t-\sigma)
\end{array}\right]\right\|_{p} \\
& \left.\leq \mu_{p}\left(\begin{array}{cc}
(-C-R) & 0 \\
0 & (-D-S)
\end{array}\right]\right)\|\Lambda(t)\|_{p}+\max \left(K\|W\|_{p}, L\|H\|_{p}\right)\|\Lambda(t-\theta)\|_{p} \\
& \leq \mu_{p}\left(\begin{array}{cc}
(-C-R) & 0 \\
0 & (-D-S)
\end{array}\right] V(t)+\max \left(K\|W\|_{p}, L\|H\|_{p}\right) \sup _{t-\theta \leq s \leq t} V(s)
\end{aligned}
$$

where $\theta=\max (\tau, \sigma)$.

Then based on the definition of upper right Dini derivative, from (9) we have

$$
D^{+} V(t) \leq \mu_{p}\left(\left[\begin{array}{cc}
(-C-R) & 0 \\
0 & (-D-S)
\end{array}\right]\right) V(t)+\max \left(K\|W\|_{p}, L\|H\|_{p}\right) \sup _{t-\theta \leq s \leq t} V(s)
$$

Let $k_{1}=-\mu_{p}\left(\left[\begin{array}{cc}(-C-R) & 0 \\ 0 & (-D-S)\end{array}\right]\right)$ and $k_{2}=\max \left(K\|W\|_{p}, L\|H\|_{p}\right)$. From the condition (5), we obtain $k_{1}>k_{2}>0$. By Lemma 1 , it can be shown that

$$
V(t) \leq \sup _{t_{0}-\theta \leq s \leq t_{0}} V(s) e^{-\lambda\left(t-t_{0}\right)}
$$

where $\lambda$ is the unique positive solution of

$$
\lambda=k_{1}-k_{2} e^{\lambda \theta}=-\mu_{p}\left(\begin{array}{cc}
(-C-R) & 0 \\
0 & (-D-S)
\end{array}\right]-\max \left(K\|W\|_{p}, L\|H\|_{p}\right) e^{\lambda \theta}
$$

Therefore, $V(t)$ converges exponentially to zero with a convergence rate of $\lambda$, or equivalently, every trajectory of system (3) will converge exponentially toward $[0,0]^{T}$ with a convergence rate of $\lambda$. Thus, we complete the proof.

\section{Numerical example}

In this section, we shall give an illustrate example to show the effectiveness of our theoretical results obtained in the previous sections.

Example 1 Consider the following delayed BAM neural networks with

$$
\begin{aligned}
& \tau=0.2, \sigma=0.3, f(y)=\frac{3}{4} y, g(x)=x . \\
& \qquad\left\{\begin{array}{l}
\dot{x}(t)=-C x(t)+W f(y(t-\tau))+I, \\
\dot{y}(t)=-D y(t)+H g(x(t-\sigma))+J,
\end{array}\right.
\end{aligned}
$$


where

$$
C=D=\left[\begin{array}{ll}
1 & 0 \\
0 & 1
\end{array}\right], \quad W=\left[\begin{array}{cc}
1.2 & -0.2 \\
-0.2 & 1.1
\end{array}\right], \quad H=\left[\begin{array}{cc}
0.8 & -0.35 \\
-0.35 & 1.1
\end{array}\right], \quad I=J=\left[\begin{array}{l}
0 \\
0
\end{array}\right] \text {. }
$$

This system satisfies Assumption 1, and it can be easily verified that $K=\frac{3}{4}, L=1$.

We chose the initial conditions of system (13) as

$$
\left\{\begin{array}{l}
\varphi_{x 1}(t)=0.1, \varphi_{x 2}(t)=0.2, t \in[-0.3,0), \\
\varphi_{y 1}(t)=0.3, \varphi_{y 2}(t)=0.4, t \in[-0.2,0) .
\end{array}\right.
$$

According to the above discussion, for the global exponential stability, we consider the following system with $r(t)=R x(t), s(t)=S y(t)$ :

$\left\{\begin{array}{l}\dot{x}(t)=-C x(t)+W f(y(t-\tau))-r(t), \\ \dot{y}(t)=-D y(t)+H g(x(t-\sigma))-s(t)\end{array}\right.$

Based on Theorem 1, if the controller gain matrices $R, S$ satisfy

$\left.-\mu_{2}\left[\begin{array}{cc}(-C-R) & 0 \\ 0 & (-D-S)\end{array}\right]\right)>\max \left(K\|W\|_{2}, L\|H\|_{2}\right)=1.3308$,

the equilibrium point of system (14) is globally exponentially stable.

Here, we select the controller gain matrices $R, S$ as:

$R_{1}=\left[\begin{array}{ll}0.8 & 0.2 \\ 0.2 & 0.6\end{array}\right], \quad S_{1}=\left[\begin{array}{ll}0.6 & 0.1 \\ 0.1 & 0.5\end{array}\right]$.

Then, with calculation by MATLAB software, we obtain

$\left.-\mu_{2}\left(\begin{array}{cc}\left(-C-R_{1}\right) & 0 \\ 0 & \left(-D-S_{1}\right)\end{array}\right]\right)=1.4382>1.3308$.

Thus, by Theorem 1, we can conclude that the equilibrium point of system (14) is globally exponentially stable. This statement is well supported by the state trajectories of variables $x_{i}(t), y_{j}(t), i=j=1,2$ of system (14) with controller gain matrices $R_{1}, S_{1}$, which are depicted in Fig. 1.

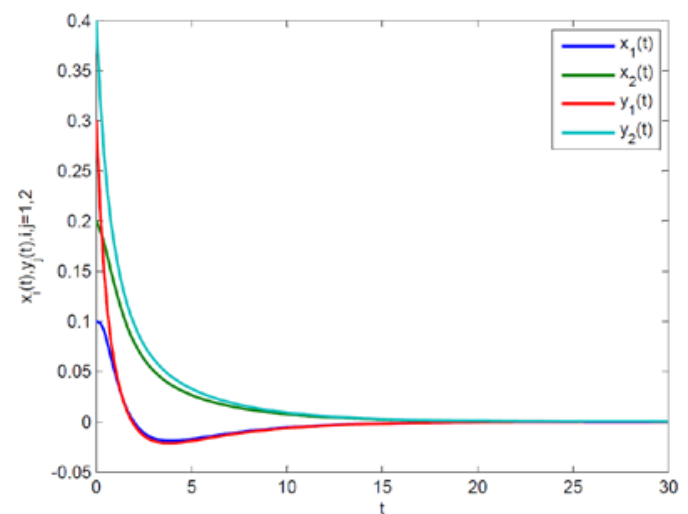

Figure1. state trajectories of variables $x_{i}(t), y_{j}(t), i=j=1,2$ of system (14) with controller gain matrices $R_{1}, S_{1}$

Different from the most previous results in which symmetry is required for the controller gain matrices, neither symmetry nor positive (negative) definiteness of the controller gain matrices are required in the results of our paper. In order to illustrate this, we select the controller gain matrices $R, S$ as:

$$
R_{2}=\left[\begin{array}{cc}
3 & -1 \\
-2 & 5
\end{array}\right], \quad S_{2}=\left[\begin{array}{cc}
4 & 0 \\
-1 & 2
\end{array}\right],
$$

Then, with calculation by MATLAB software, we obtain 


$$
-\mu_{2}\left(\left[\begin{array}{cc}
\left(-C-R_{2}\right) & 0 \\
0 & \left(-D-S_{2}\right)
\end{array}\right]\right)=2.4008>1.3308
$$

Thus, by Theorem 1, in this case we also can conclude that the equilibrium point of system (14) is globally exponentially. It shows that our method is less restrictive and more general. Figure 2 depicts the state trajectories of variables $x_{i}(t), y_{j}(t), i=j=1,2$ of system (14) with controller gain matrices $R_{2}, S_{2}$, which supports the above statement well.

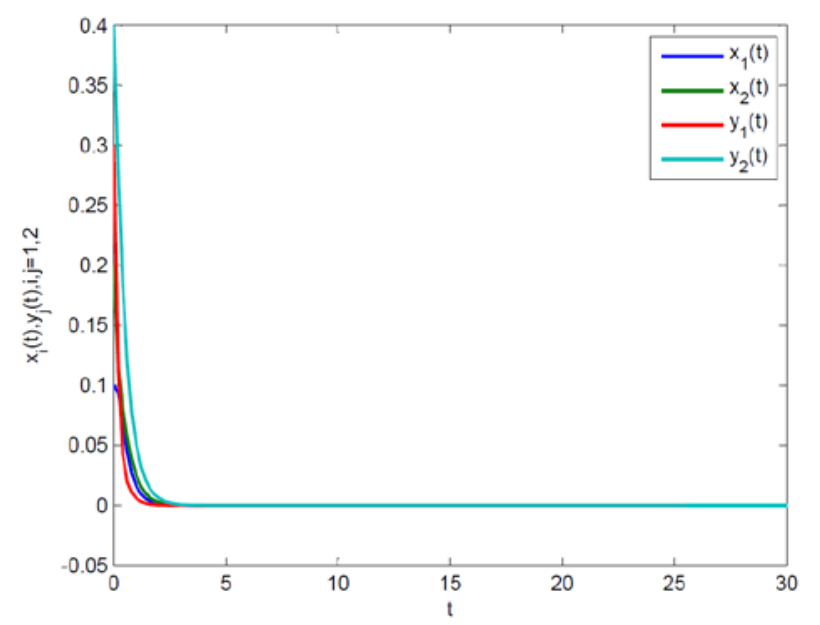

Figure 2. state trajectories of variables $x_{i}(t), y_{j}(t), i=j=1,2$ of system (14) with controller gain matrices $R_{2}, S_{2}$

\section{Conclusion}

This paper has presented a novel method to study the exponential stabilization of a class of BAM neural networks with time delays. Based on the Lyapunov stability method, we obtain several sufficient conditions for the equilibrium point to be globally exponentially stable using a novel method -matrix measure approach. Neither symmetry nor positive (negative) definiteness of the controller gain matrices are required and the proposed conditions are independent of the delay parameters. So, our conditions are less restrictive and more general. A numerical example is provided to illustrate the effectiveness and feasibility of the derived results and the novel method.

\section{References}

[1] Kosko, B., “Adaptive bidirectional associative memories.” Applied optics, vol. 26(23), 1987, pp. 4947-4960.

[2] Kosko, B., "Bidirectional associative memories.” Systems, Man and Cybernetics, IEEE Transactions on, vol. 18(1), 1988, pp. 49-60.

[3] Liu, G., Yang, S.X. and Fu, W., "New robust stability of uncertain neutral-type neural networks with discrete interval and distributed time-varying delays.” Journal of Computers, vol. 7(1), 2012, pp. 264-271.

[4] Lakshmanan, S., et al., "Stability criteria for BAM neural networks with leakage delays and probabilistic time-varying delays.” Applied Mathematics and Computation, vol. 219(17), 2013,pp. 9408-9423.

[5] Wang, M., Teng, J. F, Liu, Erlin, “Global exponential synchronization of delayed BAM neural networks.” Journal of Networks, vol. 9(5), 2014, pp.1354-1360.

[6] Wei, X. and Qiu, Z., "Anti-periodic solutions for BAM neural networks with time delays.” Applied Mathematics and Computation, vol. 221, 2013, pp. 221-229. 
[7] Feng, C. and Lin, Y., "A new result of periodic oscillations for a six-neuron BAM neural network model,” International Conference on Intelligent Computing. Springer, 2013, pp.19-24.

[8] He, X., et al., “A recurrent neural network for solving bilevel linear programming problem.” Neural Networks and Learning Systems, IEEE Transactions on, vol. 25(4), 2014, pp. 824-830.

[9] He, X., et al., "Neural network for solving convex quadratic bilevel programming problems." Neural Networks, vol. 51, 2014, pp. 17-25.

[10] He, X., et al., "Codimension two bifurcation in a delayed neural network with unidirectional coupling.” Nonlinear Analysis: Real World Applications, vol. 14(2), 2013, pp. 1191-1202.

[11] Xiao M., Zheng W. X. and Cao, J. D., "Bifurcation and control in a neural network with small and large delays,” Neural Networks, vol. 44, 2013, pp. 132-142.

[12] He, W., Cao, J. D., "Exponential synchronization of chaotic neural networks: a matrix measure approach.” Nonlinear Dynamics. vol. 55, 2009, pp. 55-65.

[13] Cao, J D, W.Y, Matrix measure strategies for stability and synchronization of inertial BAM neural network with time delays. Neural Networks, vol. 53, 2014, pp.165-172.

[14] Li, N., Cao, J. D, Intermittent control on switched networks viaw-matrix measure method. Nonlinear Dynamics. vol. 77, 2014, pp. 1363-1375.

[15] Li, Y, Li, C. D, Matrix measure strategies for stabilization and synchronization of delayed BAM neural networks, Nonlinear Dynamics, vol. 84(3), 2016, pp.1759-1770.

[16] Vidyasagar, M., Nonlinear systems analysis. Englewood Cliff. 1993, New Jersey: PrenticeHall.

[17] $\mathrm{Xu}$, J., et al., Absolute exponential stability of recurrent neural networks with generalized activation function. Neural Networks, IEEE Transactions on, vol. 19(6), 2008, pp. 1075-1089. 